\title{
A Discrete Channel Model for Capturing Memory and Soft-Decision Information: A Capacity Study
}

\author{
Cecilio Pimentel \\ Department of Electronics and Systems \\ Federal University of Pernambuco \\ Recife, PE, 50711-970, Brazil \\ Email: cecilio@ufpe.br
}

\author{
Fady Alajaji \\ Department of Mathematics and Statistics \\ Queen's University \\ Kingston, ON K7L 3N6, Canada \\ Email: fady@mast.queensu.ca
}

\begin{abstract}
A discrete (binary-input $2^{\mathrm{q}}$-ary output) communication channel with memory is introduced with the objective to judiciously capture both the statistical memory and the soft-decision information of time-correlated fading channels modulated via binary phase-shift keying and coherently demodulated with an output quantizer of resolution $q$. It is shown that the discrete channel can be explicitly described in terms of its binary input process and a $2^{\mathrm{q}}$-ary noise process. It is also shown that the channel is symmetric and admits a simple expression for its capacity when its noise is stationary ergodic. The $2^{\mathrm{q}}$-ary noise process is next modeled via a generalized version of the recently studied binary queue-based channel (2007) to produce a mathematically tractable stationary ergodic M'th order Markovian noise source with $2^{q}+2$ parameters. Numerical results indicate that the capacity of the discrete channel with $q=2,3$ is substantially improved over the cases of perfect channel interleaving (which yields an equivalent memoryless channel) and of hard-decision demodulation $(q=1)$. These results point to potentially large performance gains achievable by designing coding schemes for this discrete channel that exploit both its memory and softdecision information, as opposed to ignoring either of them.
\end{abstract}

\section{INTRODUCTION}

Wireless communication channels undergo time-varying multipath fading that is modeled as a time-correlated random process. Due to the statistical dependence of successive fading samples, the channel exhibits memory. The development of iterative decoding schemes for codes (such as low-density parity-check and turbo codes) that achieve the capacity limit of memoryless channels, when they operate on binary channels with memory, was considered in [1]-[3]. These works assume that the discrete channel (from the input of the modulator to the output of the hard-quantized demodulator) is modeled as a binary finite state Markov channel (FSMC) model and incorporate the FSMC structure in the decoding process in order to exploit the channel statistical memory. Significant performance gain is reported relative to traditional schemes that ignore the channel memory via perfect interleaving. In this latter case, the achievable rates are smaller than those of the original channel, since it is well known that memory increases capacity for a wide class of information stable channels (e.g., see [4], [5]).

Binary FSMC models that accurately approximate harddecision demodulated correlated Rayleigh and Rician flat

This work was supported in part by NSERC of Canada and CNPq of Brazil. fading channels were proposed in [6]-[9]. Besides the main interest in binary channels, information-theoretic studies reveal that soft-decision information can significantly increase the capacity for several classes of channels, including additive white Gaussian noise (AWGN) channels [10], [11], memoryless (fully interleaved) Rayleigh fading channels [12], additive colored Gaussian noise channels and intersymbol interference channels [10]. This work aims at developing simple nonbinary-output FSMC models for time-correlated flat fading channels that capture both their memory and their soft-decision information. The new models may be used in designing new coding/decoding schemes for soft-decision demodulated channels with memory that result in superior performance over systems that ignore the channel's memory (via interleaving) and/or soft-decision information (via hard demodulation).

We consider a discrete channel composed of a binary phase-shift keying (BPSK) modulator, a time-correlated flat Rayleigh fading channel, and a $q$-bit soft-quantized coherent demodulator. Motivated by the results in [11], [12], where it is shown that, for memoryless channels, important capacity gains are achieved over the hard-decision case $(q=1)$ with small values of $q$, we consider $q=2,3$.

We first describe the binary-input discrete correlated Rayleigh fading communication channel with soft-decision demodulation of resolution $q$ and show that there exists a $2^{q}$ ary noise process (independent from the input process) in such a way that the $2^{q}$-ary output process is written as an explicit function of the binary input and noise process. We refer to this binary-input discrete channel by the non-binary noise discrete channel (NBNDC). Next, we derive a formula as well as simple (numerically computable) upper and lower bounds for the capacity of this channel when its noise is stationary and ergodic (which corresponds to a stationary ergodic underlying fading process). We next model the noise process of the NBNDC via a $2^{q}$-ary Markov process generated by modifying the recently introduced binary queue-based noise process [5]. The resulting non-binary queue-based $(\mathrm{QB})$ noise source is an $M$ 'th order Markovian stationary ergodic process with $2^{q}+2$ independent parameters. Closed-form expressions for several statistics and the entropy rate of the QB noise process are established. We then conduct a numerical study to evaluate the effect of the model parameters (such as the demodulator 
resolution $q$, noise memory order $M$, and noise correlation coefficient) on the capacity of the NBNDC with QB noise. In related work, the modeling of non-binary channels via hidden Markov models has been considered in [13], [14]. However, unlike what is herein developed, these works do not provide explicit expressions for the channel statistics and capacity and are hence less amenable for tractable mathematical analysis.

\section{The Non-Binary Noise Discrete Channel Model}

\section{A. Discrete Fading Channel with Soft-Decision Demodulation}

We consider a discrete fading channel (DFC) composed of a binary BPSK modulator, a time-correlated flat Rayleigh fading channel with AWGN, and a $q$-bit soft-quantized coherent demodulator. We define the input and output alphabets of the discrete channel by $\mathcal{X}=\{0,1\}, \mathcal{Y}=\left\{0,1, \cdots, 2^{q}-1\right\}$, respectively. The complex envelope of the fading process, $\tilde{G}(t)$, is a zero-mean complex wide-sense stationary Gaussian process with a known covariance function. The sample of the fading envelope at the $k$ th signaling interval, $A_{k}=|\tilde{G}(k T)|$, where $T$ is the symbol interval, has the Rayleigh probability density function with a unit second moment. Let $\left\{X_{k}\right\}$, $X_{k} \in \mathcal{X}, k=1,2, \cdots$, be the input process to the discrete channel. The sampled received symbol at the output of the matched filter at the $k$ th signaling interval is written as

$$
R_{k}=\sqrt{E_{s}} A_{k} S_{k}+N_{k}, \quad k=1,2, \cdots
$$

where $\left\{S_{k}\right\}=\left\{\left(2 X_{k}-1\right)\right\}, E_{s}$ is the energy of the transmitted signal, $\left\{N_{k}\right\}$ is a sequence of independent and identically distributed zero-mean Gaussian random variables with variance $N_{0} / 2,\left\{A_{k}\right\}$ is a stationary time-correlated Rayleigh process. The processes $\left\{A_{k}\right\}$ and $\left\{N_{k}\right\}$ are independent of each other and of the input process. The random variable $R_{k}$ is demodulated via a $q$-bit uniform scalar quantizer to yield the discrete channel output $Y_{k} \in \mathcal{Y}$ as follows

$$
Y_{k}=j, \quad \text { if } R_{k} \in\left(T_{j-1}^{\prime}, T_{j}^{\prime}\right)
$$

for $j \in \mathcal{Y}$. The thresholds $T_{j}^{\prime}$ are uniformly spaced with stepsize $\Delta$, satisfying [12]

$$
T_{j}^{\prime}= \begin{cases}-\infty, & \text { if } j=-1 \\ \left(j+1-2^{q-1}\right) \Delta, & \text { if } j=0,1, \cdots, 2^{q}-2 \\ \infty, & \text { if } j=2^{q}-1 .\end{cases}
$$

Setting $\delta \triangleq \Delta / \sqrt{E_{s}}$ and $T_{j} \triangleq T_{j}^{\prime} / \sqrt{E_{s}}$ as the normalized step-size and thresholds, respectively, we can write $T_{j}=$ $\left(j+1-2^{q-1}\right) \delta$, for $j=0,1, \cdots, 2^{q}-2$. The conditional probability $q_{i, j}\left(a_{k}\right) \triangleq \operatorname{Pr}\left(Y_{k}=j \mid X_{k}=i, A_{k}=a_{k}\right)$, where $i \in \mathcal{X}, j \in \mathcal{Y}$ and $a_{k} \in[0, \infty)$, can be determined as follows:

$$
\begin{aligned}
q_{i, j} & \left(a_{k}\right)=\operatorname{Pr}\left(T_{j-1}^{\prime}<R_{k}<T_{j}^{\prime} \mid X_{k}=i, A_{k}=a_{k}\right) \\
= & \operatorname{Pr}\left(T_{j-1}-(2 i-1) a_{k}<\frac{N_{k}}{\sqrt{E_{s}}}<T_{j}-(2 i-1) a_{k}\right) \\
= & Q\left(\sqrt{2 \gamma}\left(T_{j-1}-(2 i-1) a_{k}\right)\right)- \\
& Q\left(\sqrt{2 \gamma}\left(T_{j}-(2 i-1) a_{k}\right)\right)
\end{aligned}
$$

where $\gamma=E_{s} / N_{0}$ is the signal-to-noise ratio (SNR) and $Q(x)=1 / \sqrt{2 \pi} \int_{x}^{\infty} \exp \left\{-t^{2} / 2\right\} d t$ is the Gaussian $Q$ function. Due to the symmetry of the BPSK constellation and the quantizer thresholds, we observe from (1) that $q_{i, j}\left(a_{k}\right)=$ $q_{1-i, 2^{q}-1-j}\left(a_{k}\right)$. We may also write

$$
q_{i, j}\left(a_{k}\right)=q_{0, \frac{j-(2 q-1) i}{(-1)^{i}}}\left(a_{k}\right)
$$

for $i \in \mathcal{X}, j \in \mathcal{Y}$. For integer $n \geq 1$, let $\operatorname{Pr}\left(y^{n} \mid x^{n}, a^{n}\right) \triangleq$ $\operatorname{Pr}\left(Y_{1}=y_{1}, \ldots, Y_{n}=y_{n} \mid X_{1}=x_{1}, \ldots, X_{n}=x_{n}, A_{1}=\right.$ $\left.a_{1}, \cdots A_{n}=a_{n}\right)$. Thus

$$
\operatorname{Pr}\left(y^{n} \mid x^{n}, a^{n}\right)=\prod_{k=1}^{n} q_{x_{k}, y_{k}}\left(a_{k}\right)=\prod_{k=1}^{n} q_{0, \frac{y_{k}-(2 q-1) x_{k}}{(-1)^{x_{k}}}}\left(a_{k}\right) .
$$

The DFC is thus probabilistically specified in terms of the channel block conditional probability

$$
\begin{aligned}
P_{\mathrm{DFC}}^{(n)}\left(y^{n} \mid x^{n}\right) & \triangleq \operatorname{Pr}\left(Y^{n}=y^{n} \mid X^{n}=x^{n}\right) \\
& =\mathbf{E}_{A_{1} \ldots A_{n}}\left[\prod_{k=1}^{n} q_{0, \frac{y_{k}-\left(2^{q}-1\right) x_{k}}{(-1)^{x} k}}\left(A_{k}\right)\right]
\end{aligned}
$$

where $y^{n}=\left(y_{1}, \cdots, y_{n}\right), x^{n}=\left(x_{1}, \cdots, x_{n}\right)$ and $\mathbf{E}_{X}[\cdot]$ denotes expectation with respect to the random variable $X$. For $n=1$, a closed-form expression for $P_{\mathrm{DFC}}^{(1)}(j), j \in \mathcal{Y}$, is given by [19]

$$
P_{\mathrm{DFC}}^{(1)}(j)=m\left(-T_{j-1}\right)-m\left(-T_{j}\right)
$$

where

$$
m\left(T_{j}\right)=1-Q\left(T_{j} \sqrt{2 \gamma}\right)-\frac{\left[1-Q\left(\frac{T_{j} \sqrt{2 \gamma}}{\sqrt{\frac{1}{\gamma}+1}}\right)\right] e^{-\frac{T_{j}^{2}}{\left(\frac{1}{\gamma}+1\right)}}}{\sqrt{\frac{1}{\gamma}+1}} .
$$

The expected value in (2) can be directly calculated for $n \leq 3$ since the joint probability density function of arbitrarily correlated Rayleigh and Rician random variables is only known in closed form for $n \leq 3$ (e.g., see [15], [16]); for $n>3$, (2) can however be determined via simulations.

We next provide an alternative representation for the DFC.

\section{B. Alternative DFC Model: The Non-Binary Noise Channel}

It is often convenient to express the channel output process $\left\{Y_{k}\right\}$ as an explicit function of the input process $\left\{X_{k}\right\}$ and a noise process $\left\{Z_{k}\right\}$, where $\left\{Z_{k}\right\}$ and $\left\{X_{k}\right\}$ are independent of each other (e.g., see [18, p. 183]). In this case, one can model the noise process via a finite-state Markov model which is an important analytical tool for coding design and performance evaluation for channels with memory.

Consider now the following non-binary noise discrete channel (NBNDC)

$$
Y_{k}=\left(2^{q}-1\right) X_{k}+(-1)^{X_{k}} Z_{k}
$$

for $k=1,2, \cdots$. We assume that the noise process $\left\{Z_{k}\right\}$ in (4) is independent of the input $\left\{X_{k}\right\}$ and is governed by the $n$ fold distribution $P_{\mathrm{NBNDC}}^{(n)}\left(z^{n}\right) \triangleq P_{\mathrm{NBNDC}}^{(n)}\left(Z_{1}=z_{1}, \cdots, Z_{n}=z_{n}\right)$, 
for $z_{k} \in \mathcal{Y}$. It directly follows from (4) and the fact that the input and noise process are independent from each other that the NBNDC $n$-fold conditional probability is given by

$$
P_{\mathrm{NBNDC}}^{(n)}\left(y^{n} \mid x^{n}\right)=P_{\mathrm{NBNDC}}^{(n)}\left(z^{n}\right)
$$

where

$$
z_{k}=\frac{y_{k}-\left(2^{q}-1\right) x_{k}}{(-1)^{x_{k}}}, \quad k=1, \cdots, n .
$$

Now, note that if the distribution of the noise process $\left\{Z_{k}\right\}$ of the NBNDC in (5) is given by (2) for each $n$, then the DFC and the NBNDC have the same channel block conditional probability. Therefore, the NBNDC provides an alternative representation of the DFC. In the special case when $q=1$ (hard-decision demodulation), the NBNDC expression in (4) reduces to the familiar expression

$$
Y_{k}=X_{k} \oplus Z_{k}
$$

(where $\oplus$ denotes addition modulo 2 ) which is widely adopted to model binary (binary-input binary-output) additive-noise discrete channels with memory [5], [6]. Furthermore, if $\left\{Z_{k}\right\}$ is memoryless, than we obtain the memoryless binary symmetric channel (BSC) which represents the fully interleaved DFC. The channel capacity of the NBNDC with stationary ergodic noise $\left\{Z_{k}\right\}$ is studied in the next section. ${ }^{1}$

\section{Channel Capacity}

Consider the NBNDC described by (4) where the noise process $\left\{Z_{k}\right\}$ is stationary ergodic. The channel capacity, in bits per channel use, for this information stable channel is given by [17], [20]

$$
C=\lim _{n \rightarrow \infty} C^{(n)}=\sup _{n} C^{(n)}
$$

where $C^{(n)} \triangleq \max _{p\left(x^{n}\right)} \frac{1}{n} I\left(X^{n} ; Y^{n}\right)$, where the maximum is taken with respect to all input distributions $p\left(x^{n}\right)$ and $I\left(X^{n} ; Y^{n}\right)$ denotes the block mutual information between $X^{n}$ and $Y^{n} . I\left(X^{n} ; Y^{n}\right)$ can be written as follows

$$
I\left(X^{n} ; Y^{n}\right)=H\left(Y^{n}\right)-H\left(Y^{n} \mid X^{n}\right)=H\left(Y^{n}\right)-H\left(Z^{n}\right)
$$

where $H(\cdot)$ denotes entropy and the last equality follows from (4) and the independence of $X^{n}$ and $Z^{n}$. Thus

$$
C^{(n)}=\frac{1}{n}\left(\max _{p\left(x^{n}\right)}\left[H\left(Y^{n}\right)\right]-H\left(Z^{n}\right)\right) .
$$

The capacity achieving input distribution that maximizes $H\left(Y^{n}\right)$ is determined next.

Definition 1: Let $\mathcal{W}=\left\{0,1, \cdots 2^{q-1}-1\right\}$ and let $\left\{W_{k}\right\}$, $W_{k} \in \mathcal{W}$, be a process with $n$-fold probability distribution

$$
\operatorname{Pr}\left(W^{n}=w^{n}\right)=\sum_{x^{n} \in \mathcal{X}^{n}} \operatorname{Pr}\left(Z^{n}=\frac{w^{n}-\left(2^{q}-1\right) x^{n}}{(-1)^{x^{n}}}\right)
$$

\footnotetext{
${ }^{1}$ Note that the stationary noise $\left\{Z_{k}\right\}$ defined by (5) and (2) is ergodic when the covariance function of the fading process $\tilde{G}(t)$ is asymptotically vanishing (such as the Clarke covariance function).
}

where $Z^{n}=\left(w^{n}-\left(2^{q}-1\right) x^{n}\right) /(-1)^{x^{n}}$ denotes the tuple obtained from component-wise operations, i.e., $\left(Z_{1}=\left(w_{1}-\right.\right.$ $\left.\left.\left.\left(2^{q}-1\right) x_{1}\right) /(-1)^{x_{1}}, \cdots, Z_{n}=w_{n}-\left(2^{q}-1\right) x_{n}\right) /(-1)^{x_{n}}\right)$. The probability assignment (8) is valid since

$$
\begin{aligned}
1 & =\sum_{z^{n} \in \mathcal{Y}^{n}} \operatorname{Pr}\left(Z^{n}=z^{n}\right) \\
& =\sum_{w^{n} \in \mathcal{W}^{n}} \sum_{x^{n} \in \mathcal{X}^{n}} \operatorname{Pr}\left(Z^{n}=\frac{w^{n}-\left(2^{q}-1\right) x^{n}}{(-1)^{x^{n}}}\right) \\
& =\sum_{w^{n} \in \mathcal{W}^{n}} \operatorname{Pr}\left(W^{n}=w^{n}\right) .
\end{aligned}
$$

The process $\left\{W_{k}\right\}$ is stationary since $\left\{Z_{k}\right\}$ is stationary; indeed for any integer $m>0, w^{n} \in \mathcal{W}^{n}$,

$$
\begin{aligned}
\operatorname{Pr} & \left(W_{1+m}=w_{1}, \cdots, W_{n+m}=w_{n}\right) \\
= & \sum_{x^{n} \in \mathcal{X}^{n}} \operatorname{Pr}\left(Z_{1+m}=\frac{w_{1}+\left(2^{q}-1\right) x_{1}}{(-1)^{x_{1}}}, \cdots,\right. \\
& \left.Z_{n+m}=\frac{w_{n}-\left(2^{q}-1\right) x_{n}}{(-1)^{x_{n}}}\right) \\
= & \sum_{x^{n} \in \mathcal{X}^{n}} \operatorname{Pr}\left(Z_{1}=\frac{w_{1}+\left(2^{q}-1\right) x_{1}}{(-1)^{x_{1}}}, \cdots,\right. \\
& \left.Z_{n}=\frac{w_{n}-\left(2^{q}-1\right) x_{n}}{(-1)^{x_{n}}}\right) \\
= & \operatorname{Pr}\left(W_{1}=w_{1}, \cdots, W_{n}=w_{n}\right) .
\end{aligned}
$$

Example 1: Let $q=2$ and $\alpha_{j}=\operatorname{Pr}\left(Z_{k}=j\right)$. The probability distribution of $W_{1}$ is given by

$$
\left(\operatorname{Pr}\left(W_{1}=0\right), \operatorname{Pr}\left(W_{1}=1\right)\right)=\left(\alpha_{0}+\alpha_{3}, \alpha_{1}+\alpha_{2}\right) .
$$

Let $\alpha_{i, j}=\operatorname{Pr}\left(Z_{k}=i, Z_{k+1}=j\right)$. The probability distribution of $W^{2}$ is given by

$$
\begin{array}{r}
\left(\operatorname{Pr}\left(W^{2}=00\right), \operatorname{Pr}\left(W^{2}=01\right), \operatorname{Pr}\left(W^{2}=10\right), \operatorname{Pr}\left(W^{2}=11\right)\right) \\
=\quad\left(\alpha_{0,0}+\alpha_{0,3}+\alpha_{3,0}+\alpha_{3,3}, \alpha_{0,1}+\alpha_{0,2}+\alpha_{3,1}+\alpha_{3,2},\right. \\
\left.\alpha_{1,0}+\alpha_{1,3}+\alpha_{2,0}+\alpha_{2,3}, \alpha_{1,1}+\alpha_{1,2}+\alpha_{2,1}+\alpha_{2,2}\right) .
\end{array}
$$

Consider the $2^{n} \times 2^{q n}$ channel transition probability matrix $\mathbf{Q}^{n}=\left[P_{\mathrm{NBNDC}}^{(n)}\left(y^{n} \mid x^{n}\right)\right]$ corresponding to $n$ channel uses, where each row (resp. column) of $\mathbf{Q}^{n}$ is indexed by a sequence $x^{n}$ (resp. $\left.y^{n}\right)$. It can be shown that $\mathbf{Q}^{n}$ is symmetric [17] since its columns can be partitioned into $2^{(q-1) n}$ arrays, where each array is of size $2^{n} \times 2^{n}$ with the property that its columns are permutations of each other and its rows are permutations of each other [17, p. 94]. This symmetry implies that the channel capacity is achieved by a uniform input distribution [17, p. 94].

Proposition 1: The value of $H\left(Y^{n}\right)$ under a uniform input distribution over $\mathcal{X}^{n}=\{0,1\}^{n}$ is given by

$$
\max _{p\left(x^{n}\right)}\left[H\left(Y^{n}\right)\right]=n+H\left(W^{n}\right) .
$$

Proof: We need to calculate

$$
H\left(Y^{n}\right)=-\sum_{y^{n} \in \mathcal{Y}^{n}} \operatorname{Pr}\left(Y^{n}=y^{n}\right) \log _{2} \operatorname{Pr}\left(Y^{n}=y^{n}\right)
$$


for a uniform input distribution. In this case

$\operatorname{Pr}\left(Y^{n}=y^{n}\right)=\frac{1}{2^{n}} \sum_{x^{n} \in \mathcal{X}^{n}} \operatorname{Pr}\left(Z^{n}=\frac{y^{n}-\left(2^{q}-1\right) x^{n}}{(-1)^{x^{n}}}\right)$.

According to the symmetry of $\mathbf{Q}^{n}$, the probability (11) is the same for $2^{n}$ distinct values of $y^{n}$. Substituting (11) into (10) and using Definition 1, we have

$\max _{p\left(x^{n}\right)}\left[H\left(Y^{n}\right)\right]=-\sum_{w^{n} \in \mathcal{W}^{n}} \operatorname{Pr}\left(W^{n}=w^{n}\right) \log _{2}\left(\frac{\operatorname{Pr}\left(W^{n}=w^{n}\right)}{2^{n}}\right)$ and hence (9) follows.

Substituting (9) into (7) yields

$$
C^{(n)}=1+\frac{1}{n}\left[H\left(W^{n}\right)-H\left(Z^{n}\right)\right] .
$$

Corollary 1: The channel capacity (6) is given by

$$
\begin{aligned}
C & =\lim _{n \rightarrow \infty} C^{(n)} \\
& =1+\lim _{n \rightarrow \infty} \frac{1}{n}\left[H\left(W^{n}\right)-H\left(Z^{n}\right)\right] \\
& =1+\mathcal{H}(W)-\mathcal{H}(Z)
\end{aligned}
$$

in bits/channel use, where $\mathcal{H}(W) \triangleq \lim _{n \rightarrow \infty}(1 / n) H\left(W^{n}\right)$ and $\mathcal{H}(Z) \triangleq \lim _{n \rightarrow \infty}(1 / n) H\left(Z^{n}\right)$ denote the entropy rates of $\left\{W_{n}\right\}$ and $\left\{Z_{n}\right\}$, respectively.

In the case of hard-decision demodulation $(q=1)$, the entropy $H\left(W^{n}\right)=0$ for all $n$, and the expression for channel capacity in (14) reduces to the well-known formula $C=1-\mathcal{H}(Z)$ (e.g., see [5]). We can directly obtain upper and lower bounds on $C$ using (13). Since $H\left(Z^{n}\right) / n$ is decreasing in $n$ for a stationary process $\left\{Z_{n}\right\}$, we have

$$
C \leq \min \left\{1,1+\frac{1}{n} H\left(W^{n}\right)-\mathcal{H}(Z)\right\} \triangleq \bar{C}^{(n)} .
$$

Combining (15) with the lower bound $C^{(n)} \leq C$ yields

$$
C^{(n)} \leq C \leq \bar{C}^{(n)} \text {. }
$$

\section{Non-Binary Queue-BASEd Markovian NoISE}

The binary queue-based channel was recently introduced in [5] to model a binary channel with a stationary ergodic binary $M$ 'th-order additive Markov noise via a finite queue. We herein generalize the binary queue set up of [5] to produce a tractable non-binary Markovian noise model for the NBNDC. The non-binary queue-based (QB) noise process $\left\{Z_{k}\right\}_{k=1}^{\infty}, Z_{k} \in \mathcal{Y}$, is generated by slightly modifying the twoparcel procedure in [5]: given that we now operate on balls with $|\mathcal{Y}|=2^{q}$ different colors (instead of only two colors), we assume that the second parcel (the urn) contains balls labeled with symbols in $\mathcal{Y}$ satisfying the probability distribution $\left(\rho_{0}, \rho_{1}, \cdots, \rho_{2^{q}-1}\right)$; see [5] for a detailed description of the procedure. The resulting QB noise process is a stationary ergodic $M$ 'th order Markov source and has only $2^{q}+2$ independent parameters (as opposed to a general Markovian process which would require in the order of $2^{q M}$ number of parameters): the size of the queue, $M$, the probability distribution of the balls in the urn, and correlation parameters $\varepsilon$ and $\alpha$, where $0 \leq \varepsilon<1, \alpha \geq 0$. The state process $\left\{S_{k}\right\}_{k=-\infty}^{\infty}$ of the QB noise, defined by $S_{k} \triangleq\left(Z_{k}, Z_{k-1}, \cdots, Z_{k-M+1}\right)$, is a homogeneous first-order Markov process with an alphabet of size $2^{q M}$. Let $p_{\underline{i} j}$ denote the conditional probability that $S_{k}=\underline{j}$ given that $S_{k-1}=\underline{i}$, where $\underline{i}=\left(i_{0}, \cdots, i_{M-1}\right)$, $\underline{j}=\left(j_{0}, \cdots, j_{M-1}\right)$,for $i_{\ell}, j_{\ell} \in \mathcal{Y}, \ell \in 0, \cdots, M-1$. We let the rows and columns of the state transition probability matrix, denoted by $\mathbf{P}$, be indexed by the vectors $\underline{i}$ and $\underline{j}$, respectively. The $(\underline{i}, \underline{j})$ th entry of the matrix $\mathbf{P}=\left[p_{\underline{i j}}\right]$ is given by

$p_{\underline{i} \underline{j}}=\left(\sum_{\ell=0}^{M-2} \delta_{j_{0}, i_{\ell}}+\alpha \delta_{j_{0}, i_{M-1}}\right) \frac{\varepsilon}{M-1+\alpha}+(1-\varepsilon) \rho_{j_{0}}$

if $j_{\ell+1}=i_{\ell}$, for $\ell=0, \cdots M-2$, or otherwise $p_{\underline{i} j}=0$, where

$$
\delta_{i, j}= \begin{cases}1, & \text { if } i=j \\ 0, & \text { if } i \neq j\end{cases}
$$

It can be shown that the $\underline{i}$ th component of the state stationary distribution column vector $\boldsymbol{\Pi}=\left[\pi_{\underline{i}}\right]$ is given by

$$
\pi_{\underline{i}}=\frac{\prod_{\ell=0}^{2^{q}-1} \prod_{m=0}^{\xi_{\ell}-1}\left((1-\varepsilon) \rho_{\ell}+m \frac{\varepsilon}{M-1+\alpha}\right)}{\prod_{k=0}^{M-1}\left((1-\varepsilon)+k \frac{\varepsilon}{M-1+\alpha}\right)}
$$

where $\prod_{k=0}^{-1}(\cdot)=1$ and $\xi_{\ell}=\sum_{k=0}^{M-1} \delta_{i_{k}, \ell}$. Thus the QB noise block probability $P_{\mathrm{QB}}\left(z^{n}\right) \triangleq \operatorname{Pr}\left(Z^{n}=z^{n}\right)$ is as follows:

- For blocklength $n \leq M$

$$
P_{\mathrm{QB}}^{(n)}\left(z^{n}\right)=\frac{\prod_{\ell=0}^{2^{q}-1} \prod_{m=0}^{\xi_{\ell}^{\prime}-1}\left((1-\varepsilon) \rho_{\ell}+m \frac{\varepsilon}{M-1+\alpha}\right)}{\prod_{k=0}^{n-1}\left((1-\varepsilon)+k \frac{\varepsilon}{M-1+\alpha}\right)}
$$

where $\xi_{\ell}^{\prime}=\sum_{k=1}^{n} \delta_{z_{k}, \ell}$.

- For blocklength $n \geq M+1$

$$
\begin{aligned}
& P_{\mathrm{QB}}^{(n)}\left(z^{n}\right)=\prod_{i=M+1}^{n}\left[\left(\sum_{\ell=i-M+1}^{i-1} \delta_{z_{i}, z_{\ell}}+\alpha \delta_{z_{i}, z_{i-M}}\right)\right. \\
& \left.\times \frac{\varepsilon}{M-1+\alpha}+(1-\varepsilon) \rho_{z_{i}}\right] \pi_{\left(z_{1}, \cdots, z_{M}\right)}
\end{aligned}
$$

where the expression for $\pi_{\left(z_{1}, \cdots, z_{M}\right)}$ is given in (16).

The correlation coefficient for the QB noise is a non-negative quantity given by

$$
\text { Cor }=\frac{\mathbf{E}\left[Z_{k} Z_{k+1}\right]-\mathbf{E}\left[Z_{k}\right]^{2}}{\operatorname{Var}\left(Z_{k}\right)}=\frac{\frac{\varepsilon}{M-1+\alpha}}{1-(M-2+\alpha) \frac{\varepsilon}{M-1+\alpha}}
$$

where $\operatorname{Var}\left(Z_{k}\right)$ denotes the variance of $Z_{k}$. We conclude this section by establishing a closed-form expression for the entropy rate $\mathcal{H}(Z)$ of the $\mathrm{QB}$ noise. Let $w_{0}, \cdots, w_{2^{q}-1}$ be non-negative integers such that $w_{0}+\cdots+w_{2^{q}-1}=M-1$ 
and let the probability distributions $\Gamma_{i}, i=0, \cdots, 2^{q}-1$, be defined as

$$
\begin{aligned}
\Gamma_{i}= & \left(\frac{w_{0} \varepsilon}{M-1+\alpha}+(1-\varepsilon) \rho_{0}, \cdots, \frac{\left(w_{i}+\alpha\right) \varepsilon}{M-1+\alpha}+(1-\varepsilon) \rho_{i},\right. \\
& \left.\cdots, \frac{w_{2^{q}-1} \varepsilon}{M-1+\alpha}+(1-\varepsilon) \rho_{2^{q}-1}\right) .
\end{aligned}
$$

Then

$$
\begin{aligned}
& \mathcal{H}(Z)=\sum_{w_{0}}^{M-1} \cdots \sum_{w_{2} q-1}^{M-1}\left(\begin{array}{c}
M-1 \\
w_{0}, w_{1}, \cdots, w_{2^{q}-1}
\end{array}\right) \times \\
& \quad\left[L_{\left(w_{0}+1, w_{1}, \cdots, w_{2} w_{-1}\right)} H\left(\Gamma_{0}\right)+L_{\left(w_{0}, w_{1}+1, \cdots, w_{2}{ }_{-1}\right)} H\left(\Gamma_{1}\right)\right. \\
& \left.+\cdots+L_{\left(w_{0}, w_{1}, \cdots, w_{2}{ }_{-1}+1\right)} H\left(\Gamma_{2^{q}-1}\right)\right]
\end{aligned}
$$

where

$$
L_{\left(w_{0}, w_{1}, \cdots, w_{2}-1\right)}=\frac{\prod_{\ell=0}^{2^{q}-1}\left(\prod_{m=0}^{w_{\ell}-1}(1-\varepsilon) \rho_{\ell}+m \frac{\varepsilon}{M-1+\alpha}\right)}{\prod_{k=0}^{M-1}\left((1-\varepsilon)+k \frac{\varepsilon}{M-1+\alpha}\right)} .
$$

\section{CAPACITy Numerical Study}

In this section, we present numerical results to examine the behavior of the channel capacity of the NBNDC with QB noise in terms of the demodulator resolution $q$, noise memory order $M$ and correlation coefficient Cor.

Since the noise process is described in terms of $2^{q}+2$ independent parameters: $\left(\rho_{0}, \cdots, \rho_{2^{q}-1}\right), \varepsilon, \alpha$ and $M$, we fix the values of $\left(\rho_{0}, \cdots, \rho_{2^{q}-1}\right)$ so that we can assess the effects of $\alpha, M$, and $\varepsilon$ (or equivalently Cor) on the channel capacity. As the underlying physical channel is the time-correlated Rayleigh fading channel described in Section II.B, we obtain the values of $\left(\rho_{0}, \cdots, \rho_{2^{q}-1}\right)$ by setting $\rho_{j}=P_{\mathrm{DFC}}^{(1)}(j)$ for $j=0, \cdots, 2^{q}-1$, where $P_{\mathrm{DFC}}^{(1)}(j)$ is given by (3) in terms of the quantization parameters $\delta$ and $q$ and the $\operatorname{SNR} \gamma .^{2}$

We first calculate the lower bound for the capacity $C^{(n)}$ using (12) and (17) for $n$ as large as 11. One objective is to determine the optimal value of the quantization step $\delta$ (in the sense of maximizing channel capacity) under different channel conditions. Fig. 1 shows $C^{(11)}$ versus $\delta$ for $q=2$ and SNR $\gamma=5 \mathrm{~dB}$. For this figure, we selected three QB noise models with varying levels of correlation and memory. The models are QB1 $(M=2, \alpha=1.0$, Cor $=0.3)$, QB2 $(M=2, \alpha=0.5$, Cor $=0.5)$ and QB3 $(M=4, \alpha=2.0$, Cor $=0.5)$. The capacity of the DMC (binary-input $2^{q}$-output) with the same values of $q, \delta, \gamma$ is also shown as a reference. Note that the DMC can be regarded as the channel resulting when perfect interleaving is employed on the NBNDC (with $\varepsilon=0$ or Cor $=$ $0)$. We observe from the curves that capacity is maximized for

\footnotetext{
${ }^{2}$ Note that we herein choose the QB noise parameters so that only the firstorder statistics of the NBNDC and the underlying fading channel are matched (i.e., $\left.\rho_{j}=P_{\mathrm{DFC}}^{(1)}(j)\right)$. As stated in the conclusions, a systematic fitting of the QB noise parameters to the DFC (conducted, as in [7], by minimizing the Kullback-Leibler divergence rate between the DFC and the NBNDC noise sources will be undertaken in future work.
}

approximately the same value of $\delta$ for all models. Thus, the values of $\delta$ tabulated in [12] for the DMC can also be used for the NBNDC with QB noise.

The effects of the parameters $M$ and $\alpha$ on the capacity of the NBNDC with QB noise are next illustrated in Fig. 2 for quantization parameters $q=2$ and $\delta=0.44$ and SNR $\gamma=5$ $\mathrm{dB}$. The following properties were proved for the channel with binary QB noise [5]: (i) its capacity strictly increases with $\alpha$ for fixed $M \geq 2$, distribution $\left(\rho_{0}, \rho_{1}\right)$ and Cor; (ii) its capacity increases with $M$, for fixed $\left(\rho_{0}, \rho_{1}\right)$ and Cor when $\alpha \leq 1$. The curves in Fig. 2 numerically point out that these two capacity properties are also valid for the NBNDC with $2^{q}$-ary QB noise, where property (ii) holds for $\alpha$ less than a certain threshold.

Fig. 3 presents upper and lower capacity bounds versus Cor for $M=2, \alpha=0.5$ and $q=1,2,3$ with $\gamma=5 \mathrm{~dB}$, and $\delta=0.44$ when $q=2$, and $\delta=0.24$ when $q=3$. For comparison purposes, we also provide the capacity of the memoryless fading channel with unquantized output and either without channel state information at both the transmitter and the receiver (labeled DMC-noCSI) [19] or with channel state information at only the receiver (labeled DMC-CSI) [21]. These capacities represent the largest achievable rates under perfect interleaving for an unquantized Rayleigh fading channel without or with channel state information, respectively. ${ }^{3}$ We remark from the figure that quantization resolutions of only 2 or 3 bits produce important capacity gains for our channel with memory relative to the hard-quantized $(q=1)$ and fully interleaved $($ Cor $=0)$ channels. For example, when Cor $=0.3$, the capacity gain of $C^{(11)}(q=3)$ over $\bar{C}^{(11)}(q=1)$ is $18.5 \%$; it is $26.5 \%$ when we compare $C^{(11)}(q=3)$ with the $\mathrm{BSC}(\mathrm{Cor}=0, q=1)$. Furthermore, for each value of $q$, there is a minimal value of noise correlation Cor for which the capacity of the NBNDC with QB noise is larger than that of the corresponding unquantized memoryless channel. These capacity gains indicate that exploiting the noise memory is more worthwhile than ignoring it via interleaving, even with low-precision quantization (small values of $q$ ).

\section{CONCLUSIONS}

We introduced a binary-input $2^{q}$-ary output discrete channel (denoted by NBNDC) to properly represent both the statistical memory and the soft-decision information of BPSK-modulated time-correlated Rayleigh fading channels when they are coherently demodulated via a $q$-bit output quantizer. The NBNDC's output is explicitly described in terms of its binary input and a $2^{q}$-ary noise. This channel reduces to the familiar binary channel with modulo- 2 additive noise when $q=1$ (i.e., under hard-decision demodulation). We derived a simple formula (as well as computable upper and lower bounds) for its capacity under stationary ergodic noise. We also generalized the binary queue-based (QB) noise process introduced in [5] to obtain a $2^{q}$-ary QB stationary ergodic $M$ th order Markovian noise model that is mathematically tractable (being fully described

\footnotetext{
${ }^{3}$ The capacity of the DMCs (corresponding to the case Cor $=0$ ) with increasing values of $q$ converges to the value indicated by DMC-noCSI when $q \rightarrow \infty$ and $\delta \rightarrow 0$ [19].
} 
in terms of $2^{q}+2$ parameters as opposed to $2^{q M}$ parameters needed for a general non-binary $M$ 'th order Markovian source). We established closed-form analytical expressions for the statistics and the entropy rate of the $2^{q}$-ary QB noise process in terms of its $2^{q}+2$ parameters. Finally, we carried a numerical study for the capacity of our NBNDC with QB noise, revealing substantial capacity gains over the cases of perfect channel interleaving and hard-decision demodulation via the use of only 2 or 3-bit output quantization.

In future work, we plan to fit the discrete soft-quantized correlated fading channel via the NBNDC with QB noise and validate the modeling in terms of codeword error probability under error correcting codes. In light of the capacity gains shown in Section V, another interesting direction is the construction of practical high-performing codes that exploit both the channel's noise memory and soft-decision information.

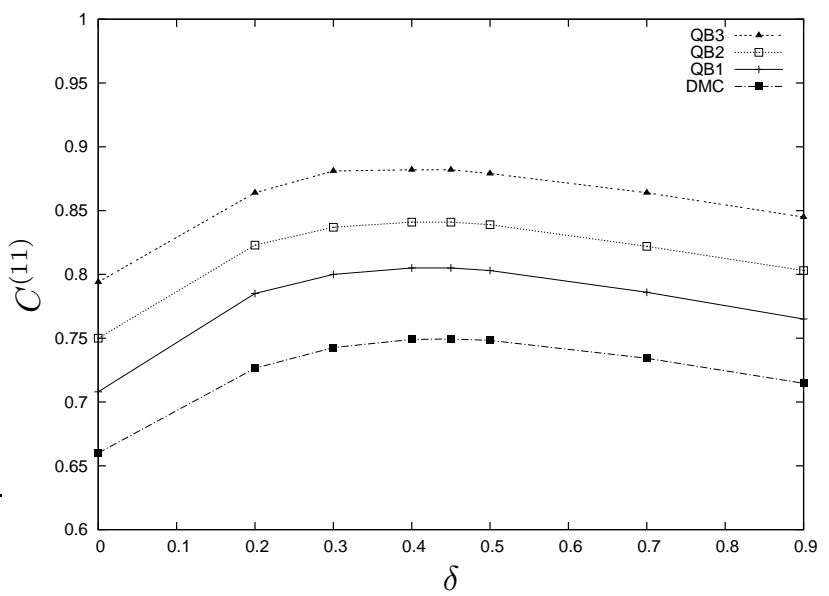

Fig. 1. $C^{(11)}$ (bits/channel use) versus $\delta$ for $q=2, \gamma=5 \mathrm{~dB}$.

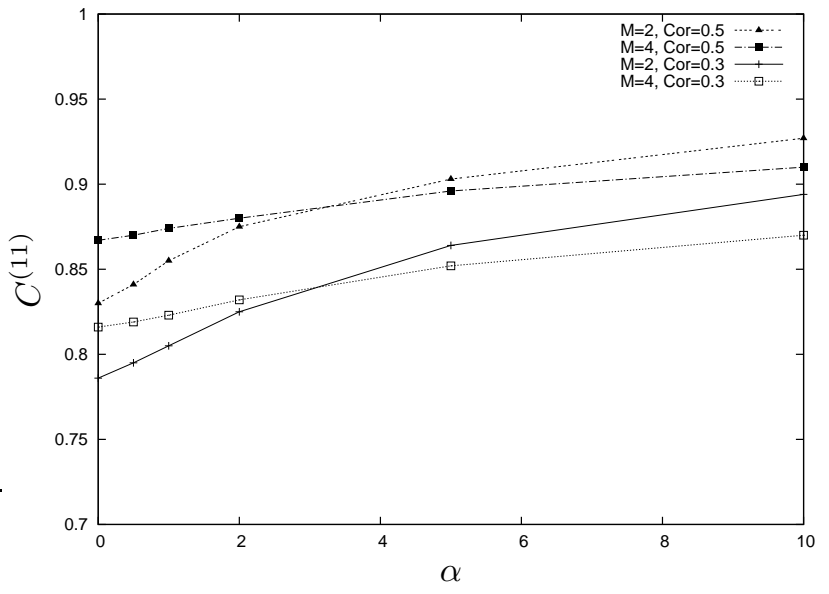

Fig. 2. $\quad C^{(11)}$ (bits/channel use) versus $\alpha$ for $q=2, \gamma=5 \mathrm{~dB}, \delta=0.44$. REFERENCES

[1] J. Garcia-Frias and J. D. Villasenor, "Turbo decoding of Gilbert-Elliott channels," IEEE Trans. Commun., vol. 50, pp. 357-363, Mar. 2002.

[2] A. W. Eckford, F. R. Kschischang and S. Pasupathy, "Analysis of lowdensity parity check codes for the Gilbert-Elliott channel," IEEE Trans. Inform. Theory, vol.51, pp. 3872-3889, Nov. 2005.

[3] C. Nicola, F. Alajaji, and T. Linder, "Decoding LDPC codes over binary channels with additive Markov noise," in Proc. Ninth Canadian Workshop Inform. Theory, Montreal, Canada, June 2005, pp. 187-190.

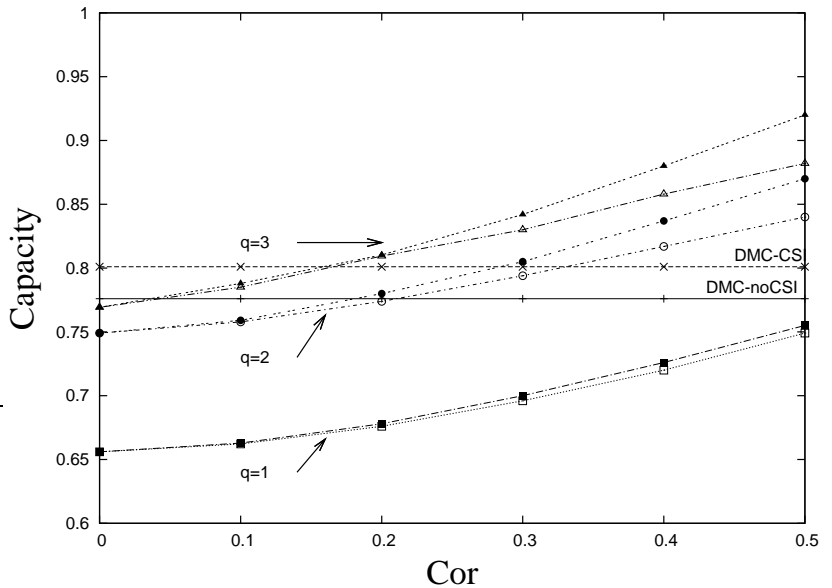

Fig. 3. Upper and lower capacity bounds (bits/channel use) versus Cor for $\gamma=5 \mathrm{~dB}, q=1,2,3$. The QB noise model has parameters $M=2, \alpha=0.5$.

[4] R. L. Dobrushin and M. S. Pinsker, "Memory increases transmission capacity", Probl. Pered. Inform., vol. 5, no. 1, pp. 94-95, 1969.

[5] L. Zhong, F. Alajaji and G. Takahara, "A binary communication channel with memory based on a finite queue," IEEE Trans. Inform. Theory, vol. 53, pp. 2815-2840, Aug. 2007.

[6] W. Turin and R. van Nobelen, "Hidden Markov modeling of flat fading channels," IEEE J. Select. Areas Commun., vol. 16, pp. 1809-1817, Dec. 1998.

[7] L. Zhong, F. Alajaji and G. Takahara, "A model for correlated Rician fading channels based on a finite queue," IEEE Trans. Veh. Technol., vol. 57, pp. 79-89, Jan. 2008.

[8] P. Sadeghi and P. Rapajic, "Capacity analysis for finite-state Markov mapping of flat-fading channels," IEEE Trans. Commun., vol. 53, pp. 833-840, May 2005.

[9] C. Pimentel and I. F. Blake, "Modeling burst channels using partitioned Fritchman's Markov models," IEEE Trans. Veh. Technol., vol. 47, pp.885-899, Aug. 1998.

[10] N. Phamdo and F. Alajaji,"Soft-decision demodulation design for COVQ over white, colored, and ISI Gaussian channels," IEEE Trans. Comm., vol. 48, pp. 1499-1506, Sept. 2000.

[11] J. Singh, O. Dabeer and U. Madhow, "Capacity of the discrete-time AWGN channel under output quantization," in Proc. IEEE Int. Symp. Inform. Theory, Toronto, July 2008, pp. 1218-1222.

[12] F. Alajaji and N. Phamdo, "Soft-decision COVQ for Rayleigh-fading channels," IEEE Commun. Letters, vol. 2, pp. 162-164, June 1998.

[13] F. Swarts and H. C. Ferreira, "Markov characterization of channels with soft decision outputs," IEEE Trans. Commun., vol. 41, pp. 678-682, May 1993.

[14] P. Kuczynski, A. Rigollé, W. H. Gerstacker, and J. B. Huber, "Hidden Markov modeling of error patterns and soft outputs for simulation of wideband CDMA transmission systems," Int. J. Electron. Commun., vol. 58, pp. 256-267, 2004

[15] Y. Chen and C. Tellambura, "Infinite series representations of the trivariate and quadrivariate Rayleigh distribution and their applications," IEEE Trans. Commun., vol. 53, pp. 2092-2101, Dec. 2005.

[16] P. Dharmawansa, N. Rajatheva and C. Tellambura, "On the trivariate Rician distribution," IEEE Trans. Commun., vol. 56, pp. 1993-1997, Dec. 2008.

[17] R. G. Gallager, Information Theory and Reliable Communication, New York: Wiley, 1968.

[18] R. W. Yeung, A First Course in Information Theory, New Jersey: Springer-Verlag, 2006.

[19] G. Taricco, "On the capacity of the binary input Gaussian and Rayleigh fading channel," Eur. Trans. Telecommun., vol. 7, pp. 201-208, Mar.Apr. 1996.

[20] S. Verdú and T. S. Han, "A general formula for channel capacity," IEEE Trans. Inform. Theory, vol. 40, pp. 1147-1157, July 1994.

[21] E. K. Hall and S. G. Wilson, "Design and analysis of Turbo codes on Rayleigh fading channels," IEEE J. Select. Areas Commun., vol. 16, pp. 160-174, Feb. 1998. 